\title{
Tollmien-Schlichting route to elastoinertial turbulence in channel flow
}

\author{
Ashwin Shekar, ${ }^{1}$ Ryan M. McMullen, ${ }^{2}$ Beverley J. McKeon $\odot,{ }^{2}$ and Michael D. Graham ${ }^{1}{ }^{1 *}$ \\ ${ }^{1}$ Department of Chemical and Biological Engineering, University of Wisconsin-Madison, \\ Madison, Wisconsin 53706, USA \\ ${ }^{2}$ Graduate Aerospace Laboratories, California Institute of Technology, Pasadena, California 91125, USA
}

(Received 20 April 2021; accepted 31 August 2021; published 27 September 2021)

\begin{abstract}
Direct simulations of two-dimensional channel flow of a viscoelastic fluid have revealed the existence of a family of Tollmien-Schlichting (TS) attractors that is nonlinearly selfsustained by viscoelasticity [Shekar et al., J. Fluid Mech. 897, A3 (2020)]. Here, we describe the evolution of this branch in parameter space and its connections to the Newtonian TS attractor and to elastoinertial turbulence (EIT). At Reynolds number Re $=3000$, there is a solution branch with TS-wave structure but which is not connected to the Newtonian solution branch. At fixed Weissenberg number, Wi, and increasing Reynolds number from 3000 to 10000 , this attractor goes from displaying a striation of weak polymer stretch localized at the critical layer to an extended sheet of very large polymer stretch. We show that this transition is directly tied to the strength of the TS critical layer fluctuations and can be attributed to a coil-stretch transition when the local Weissenberg number at the hyperbolic stagnation point of the Kelvin cat's eye structure of the TS wave exceeds $\frac{1}{2}$. At $\operatorname{Re}=10000$, unlike 3000, the Newtonian TS attractor evolves continuously into the EIT state as Wi is increased from zero to about 13. We describe how the structure of the flow and stress fields changes, highlighting in particular a "sheet-shedding" process by which the individual sheets associated with the critical layer structure break up to form the layered multisheet structure characteristic of EIT.
\end{abstract}

DOI: 10.1103/PhysRevFluids.6.093301

\section{INTRODUCTION}

The addition of minute quantities of long chain polymer molecules has tremendous effects on wall-bounded turbulence, the most dramatic and well known effect being the reduction of friction factor by levels unmatched by additive-free turbulence control schemes. The rheological drag reduction phenomenon was first discovered by Toms in the 1940s [1,2], and is sometimes referred to as the "Toms effect." This technology has found use in various applications that seek energy efficiency - transportation of oil, fracking, and heat and cooling systems, to name a few [3-5].

Accompanying the drastic reduction in friction factor is a structural change to the flow-a well known effect is the suppression by viscoelasticity of the near-wall streamwise vortices that dominate Newtonian turbulence [6,7]. A number of studies have captured this phenomena by studying the effect of viscoelasticity on three-dimensional (3D) nonlinear traveling wave solutions of the Navier-Stokes equations termed exact coherent states (ECS) [8-13]. These ECS contain the basic self-sustaining ingredients of transitional Newtonian turbulence, i.e., quasistreamwise vortices and streaks. A comprehensive review of Newtonian ECS can be found in [14]. In particular, Li and co-workers $[12,13]$ focused on one such family of ECS in channel flow and observed a weakening of structures on increasing Weissenberg number (Wi), the ratio between the polymer relaxation

*mdgraham@wisc.edu 
time scale and the flow time scale. At sufficiently high Wi, these structures are so weakened that the ECS are no longer self-sustained and lose existence. These results indicate that the near-wall self-sustaining process of Newtonian turbulence is disrupted by viscoelasticity. Recognizing that, in general, viscoelasticity is not experimentally observed to drive relaminarization, these authors suggested the possibility of new viscoelastic mechanisms for turbulence coming into existence and being unmasked as the Newtonian structure is suppressed [12].

Indeed, instead of complete relaminarization (except in narrow parameter ranges at transitional Re as detailed later), recent studies have unearthed a polymer-driven chaotic flow state dubbed elastoinertial turbulence (EIT) dominating the flow at high levels of viscoelasticity [16]. EIT was shown to display tilted multilayered sheets of polymer stretch with weak spanwise-oriented flow structures - a sharp contrast to the 3D quasistreamwise vortex structures that make up inertia-driven Newtonian turbulence. Gaining an understanding of the self-sustenance mechanisms that underlie EIT has been the focus of recent efforts. Here, we build on previous observations that tie EIT to mechanisms originating from Tollmien-Schlichting (TS) waves and uncover the underlying process that gives rise to the structures seen in EIT.

Choueiri et al. [17] experimentally examined EIT by focusing on parameter regimes close to the inception of Newtonian turbulence in pipes. At low transitional $\mathrm{Re}=3150$ and varying polymer concentration in a quasistatic way, these authors observed a complete relaminarization of Newtonian turbulence in a narrow window of concentration, followed by a reentrant transition to EIT. These observations bring to light two distinct self-sustenance mechanisms in dilute polymer solutions-one that is suppressed by viscoelasticity (Newtonian turbulence) and one that is promoted (elastoinertial turbulence).

Using computations of flowing dilute polymeric solutions in channel flow, Shekar et al. [18] shed critical insights into the viscoelastic mechanisms underlying EIT. At fixed $\mathrm{Re}=1500$ and varying $\mathrm{Wi}$, they observed a narrow zone of Wi where the only attractor was the laminar base state. This separated drag-reduced Newtonian turbulence at lower Wi and EIT at higher Wi, corroborating the experimental observations of [17]. Further, finite amplitude perturbations were required to transition to EIT, indicating a new viscoelastic bypass transition route to EIT. By analyzing the structures at EIT, they were able to uncover clues into the nature of this transition. Close to its inception, EIT in this parameter regime displayed polymer stretch fluctuations localized near the wall. To shed light on the origins of these localized fluctuations, Shekar et al. [18] used a combination of linear stability analysis and resolvent analysis, which quantifies the linear response of the system to external forcing in frequency space. In particular, they observed a clear resemblance of the EIT structure in this parameter range to the viscoelastic Tollmien-Schlichting (TS) mode, which at the chosen parameters was the slowest decaying mode from linear stability analysis. Similarly, resolvent analysis predicted strong amplification of this structure in the presence of viscoelasticity. This strong amplification implies that even very weak disturbances may be sufficient to trigger the nonlinear effects necessary to sustain EIT. The viscoelastic TS mode displays polymer stretch fluctuations that are sharply localized to critical layers, i.e., wall-normal positions near the top and bottom walls where the streamwise velocity equals the real part of the wave speed. Critical layers can be thought of as the most favorable positions for energy exchange between the mean and fluctuations, because they are the positions where both have the same speed. These results indicate a role for TS-like critical layer mechanisms at EIT. Indeed, the initial EIT computations of Samanta et al. [16], as well as followup computations [19,20], show strong localization of fluctuations in a layer near the wall. Sid et al. [21] later showed that EIT is fundamentally 2D in nature by performing simulations in 2D channel flows. Relatedly, Haward et al. [22,23] present experiments and analysis for viscoelastic flow over a wavy wall that illustrate amplification of perturbations in the critical layer.

Similar structures have been observed in a range of flow scenarios, indicating a robustness in the underlying mechanisms. Near-wall localized, nearly axisymmetric vortex and stress structures have been reported in pipe flow simulations of EIT by Lopez et al. [24]. This near-wall nature of EIT in pipes was recently corroborated in experiments by Choueiri et al. [25]. This is exactly what we would expect if critical layers are also a strong source of linear amplification 


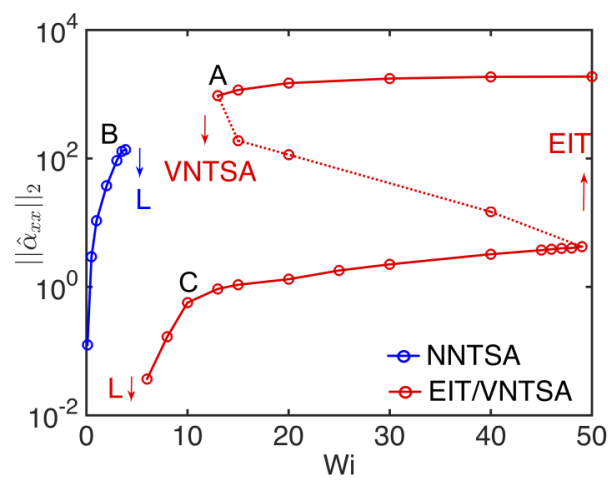

(a)

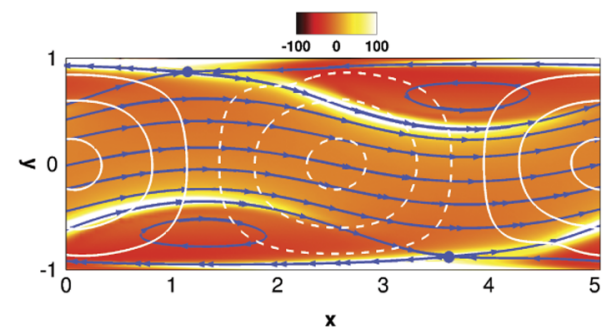

(c)

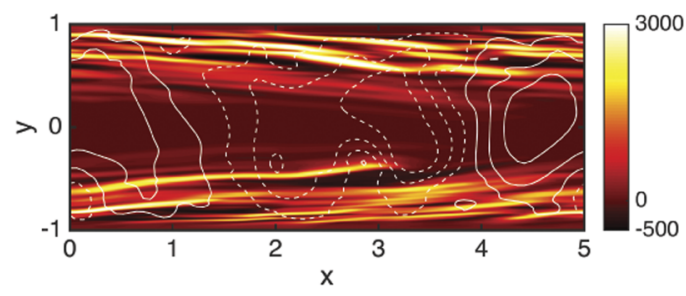

(b)

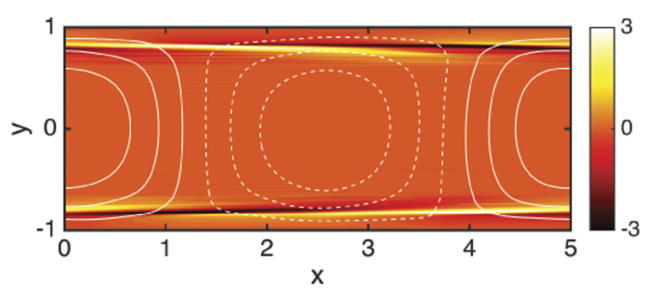

(d)

FIG. 1. (a) Time-averaged $L_{2}$ norm of $\hat{\alpha}_{x x}$ vs Wi of the three solution branches identified in [15] at $\mathrm{Re}=3000, L_{x}=5$. Snapshots corresponding to (b) 2D EIT at Wi $=13$ (point A on the bifurcation diagram), (c) NNTSA at $\mathrm{Wi}=3$ (point B), and (d) VNTSA at Wi $=10$ (point C). Shown are white contour lines of wall normal velocity, $\hat{v}$, superimposed on color contours of $x x$ component of polymer conformation tensor $\left(\hat{\alpha}_{x x}\right)$. Here ${ }^{\wedge}$ denotes deviations from laminar base state. For point B, we also show the streamlines (blue) in a reference frame moving at the wave speed and the hyperbolic stagnation points (blue dots).

in pipe flow, as they are in channel flow [26]. Indeed, Zhang [27] recently performed resolvent analysis for pipe flow in the same parameter regime, demonstrating that the most amplified mode has strong stress fluctuations localized in a critical layer near the wall, just as was found by Shekar et al. [18]. Simulations of turbulent Couette flow by Pereira et al. [28] indicate a "pseudolaminar state" with weak wall-localized fluctuations as a precursor to the emergence of EIT. Again, the structures observed were nearly 2D, with near-wall localization. Very recent computations by Zhu et al. [29] observe an intermittent process in drag-reduced turbulence involving spanwise-oriented quasi-2D structures with critical layer characteristics and 3D quasistreamwise structures. While the TS instability per se does not exist in pipe and plane Couette geometries, that fact does not preclude nonlinear mechanisms with near-wall structure coming into existence at a finite amplitude to underlie EIT in these contexts. We expand on this point below.

Given the 2D nature of EIT at transitional Re, Shekar et al. [15] studied the bifurcation scenario of 2D channel flow EIT at $\mathrm{Re}=3000$ and its ties to nonlinear self-sustaining TS waves. In that study, the domain was chosen to be five channel half-heights long, because, at this length, self-sustaining nonlinear Tollmien-Schlichting waves exist in the Newtonian case for $\mathrm{Re} \gtrsim 2800$ [30]. Some key results of this study that form the basis for the present work are described below and summarized in Fig. 1. At this parameter set, 2D EIT [upper branch of the red curve on the bifurcation diagram of Fig. 1(a)] comes into existence at $\mathrm{Wi} \approx 13$. A snapshot of the $x x$ component of the polymer stretch, displaying tilted, multilayered sheets at EIT, is shown in Fig. 1(b). These authors identify 
two separate branches of attractors based on the TS mode. The first [solid blue curve on Fig. 1(a)] originates with the Newtonian nonlinear traveling wave solution and loses existence above $\mathrm{Wi} \approx 4$. This solution branch is termed the Newtonian nonlinear Tollmien-Schlichting attractor (NNTSA); it develops a sheet of high polymer stretch that starts out near the wall and arches away from it as shown in Fig. 1(c). This structure originates with the Kelvin cat's eye structure of TS waves, specifically its near-wall hyperbolic stagnation points [blue circles in Fig. 1(c)]. These observations demonstrate the capability of nonlinear TS critical layer mechanisms in generating sheets of polymer stretch.

The second solution branch (lower solid-red curve) with TS-like structure comes into existence at finite amplitude when $\mathrm{Wi} \approx 6$. This branch, denoted the viscoelastic nonlinear Tollmien-Schlichting attractor (VNTSA), was first found by starting with an initial condition at EIT, then lowering Wi below the threshold value $\mathrm{Wi} \approx 13$ noted above. It has extremely weak fluctuations and seems likely to be related to the pseudolaminar state observed by Pereira et al. [28]. This state displays striations of polymer stretch localized at the critical layer [Fig. 1(d)] and is structurally very similar to the linear TS mode at the same value of $\mathrm{Wi}$, though it displays weakly chaotic rather than purely time-periodic (traveling wave) fluctuations. The existence of such a state indicates the presence of a nonlinear viscoelastic mechanism for self-sustenance of TS structure, an observation that has important implications in the broader context of other flow scenarios such as pipe flow, where no linear TS instability exists. On increasing Wi, the VNTSA develops multilayered striations of polymer stretch before joining up with 2D EIT through an unstable branch indicated by the red-dashed line in Fig. 1(a).

In the present work, we elaborate on the relationship between nonlinear Tollmien-Schlichting structures and elastoinertial turbulence. In Sec. III A, we describe the evolution of the VNTSA as Re increases from 3000 to 10000 . We detail observations of a coil-stretch transition, with a jump in polymer stretching - almost two orders of magnitude - when the local Wi at the hyperbolic stagnation point of the Kelvin cat's eye structure crosses $\frac{1}{2}$. Accompanying this transition is a structural change from localized striations to extended sheets. These form the necessary ingredients for a process we coin the "sheet shedding process" responsible for generating multilayered sheets of polymer stretch, a key characteristic of EIT. We describe this process at Re $=10000$ and trace its origins in Sec. III B. At this Reynolds number, the Newtonian nonlinear Tollmien-Schlichting solution is directly connected to EIT as Wi increases. Interesting intermittent dynamics occur along this branch, as described in Sec. III C. In Sec. IIID, we show the robustness of these observations with respect to polymer extensibility and domain size. Finally, in Sec. III E we discuss the broader context of our results.

\section{FORMULATION}

This study focuses on two-dimensional pressure-driven channel flow with constant mass flux. The $x$ and $y$ axes are aligned with the streamwise and wall-normal directions, respectively. Lengths are scaled by the half channel height $l$, so the dimensionless channel height $L_{y}=2$. The domain is periodic in $x$ with length $L_{x}$. Velocity $\boldsymbol{v}$ is scaled with the Newtonian laminar centerline velocity $U$, time $t$ with $l / U$, and pressure $p$ with $\rho U^{2}$, where $\rho$ is the fluid density. The polymer stress tensor $\boldsymbol{\tau}_{p}$ is related to the polymer conformation tensor $\boldsymbol{\alpha}$ through the FENE-P constitutive relation, which models each polymer molecule as a pair of beads connected by a nonlinear spring with maximum extensibility $b$.

We solve the momentum, continuity, and FENE-P equations:

$$
\begin{gathered}
\frac{\partial \boldsymbol{v}}{\partial t}+\boldsymbol{v} \cdot \nabla \boldsymbol{v}=-\nabla p+\frac{\beta}{\operatorname{Re}} \nabla^{2} \boldsymbol{v}+\frac{(1-\beta)}{\operatorname{ReWi}}\left(\nabla \cdot \tau_{\mathrm{p}}\right), \\
\nabla \cdot \boldsymbol{v}=0,
\end{gathered}
$$




$$
\begin{gathered}
\boldsymbol{\tau}_{p}=\frac{\boldsymbol{\alpha}}{1-\frac{\operatorname{tr}(\boldsymbol{\alpha})}{b}}-\boldsymbol{I}, \\
\frac{\partial \boldsymbol{\alpha}}{\partial t}+\boldsymbol{v} \cdot \nabla \boldsymbol{\alpha}-\boldsymbol{\alpha} \cdot \nabla \boldsymbol{v}-(\boldsymbol{\alpha} \cdot \nabla \boldsymbol{v})^{\mathrm{T}}=\frac{-1}{\mathrm{Wi}} \boldsymbol{\tau}_{p} .
\end{gathered}
$$

Here $\operatorname{Re}=\rho U l /\left(\eta_{\mathrm{s}}+\eta_{\mathrm{p}}\right)$, where $\eta_{s}$ and $\eta_{p}$ are the solvent and polymer contributions to the zero-shear rate viscosity. The viscosity ratio $\beta=\eta_{\mathrm{s}} /\left(\eta_{\mathrm{s}}+\eta_{\mathrm{p}}\right)$. We fix $\beta=0.97$ and $b=6400$ for all results except in the last section where we use $b=10^{5}$. Since $1-\beta$ is proportional to polymer concentration and $b$ to the number of monomer units, these parameters correspond to a dilute solution of a high molecular weight polymer. The Weissenberg number $\mathrm{Wi}=\lambda U / l$, where $\lambda$ is the polymer relaxation time.

For the nonlinear direct numerical simulations (DNS) described below, a finite difference scheme and a fractional time step method were adopted for integrating the momentum equation. Secondorder central differences were used for spatial discretization and second-order Adams-Bashforth and Crank-Nicolson methods were used for time-integration of the convection and diffusion terms, respectively. The FENE-P equation was discretized using a high resolution central difference scheme, the second-order Kurganov-Tadmor scheme [31-33], and, as in [33], time integrated with forward Euler. No artificial diffusion was applied. A time step of 0.001 was used, corresponding to a Courant number $\sim 10^{-2}$. Further details and validation cases can be found in [34] and [35]. Resolution tests were performed to ensure convergence of statistics. A typical resolution for the following results is $\left(N_{x}, N_{y}\right)=(131,602)$. This resolution used was based on mesh convergence results at the highest Re and Wi (10000 and 13) considered here. When the resolution was increased to $\left(N_{x}, N_{y}\right)=(181,702)$, the mean polymer stretch deviations from the laminar base state change by less than $1 \%$. We also perform computations in the shift-reflect symmetric subspace innate to the TS solution family. These computations are done by simulating half the domain and applying the following symmetry to generate the fields in the other half:

$$
\left[\begin{array}{llllll}
u & v & \alpha_{x x} & \alpha_{y x} & \alpha_{y y} & \alpha_{z z}
\end{array}\right]\left(x+L_{x} / 2, y, t\right)=\left[\begin{array}{llllll}
u & -v & \alpha_{x x} & -\alpha_{y x} & \alpha_{y y} & \alpha_{z z}
\end{array}\right](x,-y, t) .
$$

\section{RESULTS AND DISCUSSION}

\section{A. Viscoelastic Tollmien-Schlichting attractor: From $R e=3000$ to 10000}

The results presented in this section make the connection between the results of Shekar et al. [15] and the bulk of the present work, which will focus on the attractor at $\mathrm{Re}=10000$. We begin by considering the evolution of the VNTSA as Re is increased from 3000 to 10000 at fixed Wi $=10$, $L_{x}=6.2$. To find these solutions, an initial condition of the laminar state perturbed by the linear TS mode was used to land on the attractor at $\mathrm{Re}=3000$, following which the velocity and polymer stretch fields from the nonlinear TS attractor at a one Re were used as initial conditions at a higher Re to trace out the evolution of the attractor from $\operatorname{Re}=3000$ to 10000 . We then performed the same process but moving downward in Re from the solution at 10000 to verify that there is no hysteresis on this solution branch upon changing Re. Note the difference in box size from the results in [15]. In the earlier study, $L_{x}$ was chosen based on the lowest Re for existence of finite amplitude nonlinear TS waves. In contrast, in the present work, $L_{x}$ was chosen to approximately correspond to the domain size at which the linear TS instability first appears (at $\mathrm{Re}=5772$ ) [36]. This was done so that we could examine, as we do below, the behavior when the TS mode is linearly unstable to explore the relationship between the NNTSA, VNTSA, and EIT. Indeed, at the value $L_{x}=5$ chosen in the earlier work, the TS mode does not become linearly unstable at any Re.

The blue curve on Fig. 2(a) quantifies the evolution of the VNTSA in Re using the time-averaged $L_{2}$ norm of $\hat{\alpha}_{x x}$ as a measure. Unless otherwise noted, all quantities reported here are time averaged. With the attractor being weakly chaotic, at least 3000 time units (TU) of data, and in some cases up to $6000 \mathrm{TU}$, are used to get an accurate estimate of this quantity. The key observation is that, 


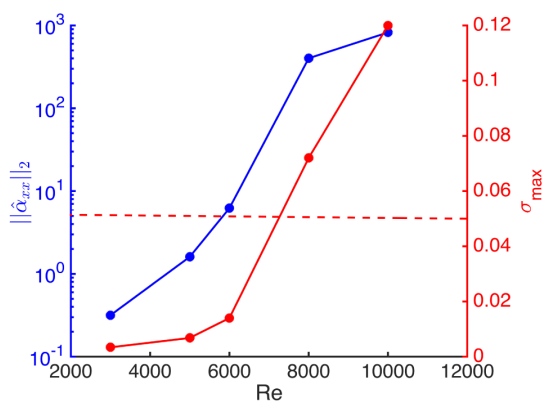

(a)

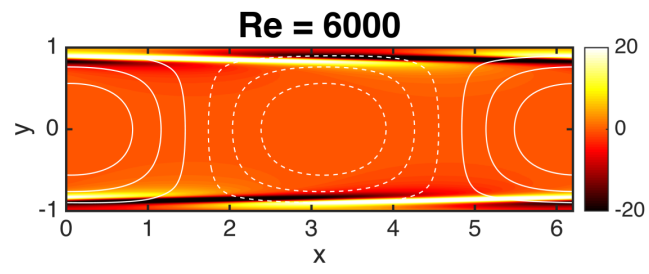

(b)

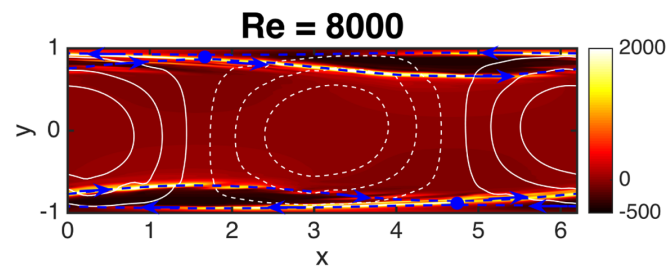

(c)

FIG. 2. (a) $L_{2}$ norm of $\hat{\alpha}_{x x}$ (blue) and corresponding mean local stretch rate at the hyperbolic stagnation point (red) along the VNTSA branch at $\mathrm{Wi}=10$. Red-dashed line corresponds to $\mathrm{Wi}_{\mathrm{loc}}=\lambda \sigma_{\max }=1 / 2$. Panels (b) and (c) are snapshots of the fluctuation structure of the solution branch at $\operatorname{Re}=6000$ and 8000 , respectively. Shown are contour lines of $\hat{v}$ superimposed on color contours of $\hat{\alpha}_{x x}$. Here^denotes deviations from laminar base state. On (c), the hyperbolic stagnation points in the traveling frame are indicated as blue dots and the streamlines attached to them as dashed curves with arrows indicating the direction of flow.

in the range $6000<\operatorname{Re}<8000,\left\|\hat{\alpha}_{x x}\right\|_{2}$ increases by several orders of magnitude. Note that, to capture this large change, a log scale is used. Figures 2(b) and 2(c) are representative snapshots of the flow and polymer conformation fields, which illustrate the structural change accompanying this transition. Throughout this manuscript, we report the deviations from the laminar state, rather than the total. Otherwise, for cases where the TS structure is weak, especially in the case of $\alpha$, it would not be discernible.

As at $\operatorname{Re}=3000$, the attractor at $\operatorname{Re}=6000$ [Fig. 2(b)] continues to display striations of weak polymer stretch fluctuations that are localized near the wall, and closely resembles the linear TS mode. Apart from the obvious quantitative differences, the attractor at 8000 [Fig. 2(c)] now displays a sheet of very strong polymer stretch emanating from the near-wall stagnation point regions. Additionally, at $\operatorname{Re}=8000$, we see that, in the region near the bottom wall where the wall-normal velocity is negative (roughly $1.6<x<4.6$ ), the sheet of high stretching seems to have broken into several smaller sheets, and similarly near the top wall where $v_{y}>0$. We elaborate below on the "sheet shedding" process that leads to this structure, and how it connects the TS structure to EIT.

To shed insights into the nature of this transition, we examine the flow kinematics more quantitatively. Aside from a very weak temporally nonperiodic component, the flow is a traveling wave displaying the characteristic Kelvin cat's eye streamlines, and the corresponding hyperbolic stagnation points, in the traveling frame. Denoting the largest eigenvalue of the velocity gradient $\nabla \mathbf{v}^{T}$ at these points as $\sigma_{\max }$, material lines near these points will stretch along the unstable direction of the stagnation point as $\exp \left(\sigma_{\max } t\right)$. Polymer molecules near these points will also stretch exponentially if $\lambda \sigma_{\max }>1 / 2$ [37,38]. Defining a local Weissenberg number $\mathrm{Wi}_{\mathrm{loc}}=\lambda \sigma_{\max }$, this criterion becomes $\mathrm{Wi}_{\text {loc }}>1 / 2$. Figure 2(a) reports estimates (red) of $\sigma_{\max }$ vs Re. Values are in the range $0.01 \lesssim \sigma_{\max } \lesssim 0.1$ (in units of $U / l$ ), indicating that $\mathrm{Wi}_{\text {loc }} \ll \mathrm{Wi}$. The origin of this difference 
in magnitude can be understood as follows: recall that the laminar velocity field is everywhere a pure shear flow, for which the straining and vortical (symmetric and antisymmetric) components of $\nabla \mathbf{v}^{T}$ are equal, both of its eigenvalues are zero, it has only one eigenvector, $[1,0]^{T}$, and material line stretching is everywhere linear in time, no matter how large Wi is. The velocity field of the VNTSA is a relatively weak perturbation away from the laminar flow and, accordingly, the eigenvalues of the velocity gradient tensor are weakly perturbed from zero: $\sigma_{\max } \ll 1$ or equivalently $\mathrm{Wi}_{\text {loc }} \ll \mathrm{Wi}$. Furthermore, at the hyberbolic stagnation points, the eigenvectors of $\nabla \mathbf{v}^{T}$ (the unstable and stable directions) are nearly aligned, because they both emerge from the single eigenvector $[1,0]^{T}$ of the degenerate pure shear case. Figure 2(c) shows the positions of the hyperbolic stagnation points (blue dots) and the streamlines emanating from these points at $\mathrm{Re}=8000$. Observe the small angle between the streamlines entering and leaving the stagnation points, as well as the sheet of highly stretched fluid oriented along the unstable direction. At $\operatorname{Re}=6000$ the angle is too small to see clearly so we opted not to show the streamlines for that case.

Since the attractors are not pure traveling waves, the estimates of $\sigma_{\max }$ in Fig. 2(a) are based on time averaging over multiple snapshots sampled over the attractor. For a given snapshot, say at $t=2000$, the fields at $t=1999$ and $t=2001$ would be used to estimate the instantaneous wave speed by calculating the streamwise distance the position of the maximum wall normal velocity moves. This wave speed is then subtracted off of the streamwise velocity to yield the velocity field in the traveling frame, from which the stagnation point positions can be identified and $\sigma_{\max }$ estimated. The drastic change between $\mathrm{Re}=6000$ and 8000 coincides with $\mathrm{Wi}_{\text {loc }}$ crossing the coil-stretch threshold $\mathrm{Wi}_{\text {loc }}=1 / 2$.

\section{B. $\operatorname{Re}=10$ 000: Sheet shedding and the evolution from TS waves to EIT}

At $\operatorname{Re}=3000$, the solution branch described in the previous section loses existence as Wi is decreased to zero. In contrast, at $\mathrm{Re}=10000$, the attractor presented in the previous section at $\mathrm{Wi}=$ 10 can be traced continuously and nonhysteretically back to the Newtonian attractor on decreasing Wi. Thus, at these conditions, the NNTSA and VNTSA are no longer distinct solution branches, so we simply refer to the TS attractor. Equally importantly, the solution at $\mathrm{Wi}=10$ evolves, with a slight further increase in Wi, to EIT. That is, at $\mathrm{Re}=10000$, the Newtonian nonlinear TollmienSchlichting solution evolves continuously to EIT as Wi increases.

Figure 3(a) shows this evolution using $\left\|\hat{\alpha}_{x x}\right\|_{2}$ as the measure. To generate the results in this section, initial conditions of the laminar base state perturbed by the linear TS mode were used to land on the nonlinear attractor at each Wi. Furthermore, a second set of runs were performed using the fields from the nonlinear attractor at a certain Wi as initial conditions at higher or lower Wi. Results on both increasing and decreasing Wi were consistent with the first set of runs, indicating the absence of hysteresis. Also shown on this plot are the standard deviations of this statistic to give a sense of the temporal intermittency of the dynamics along the branch. Here, the results in the full space (red) are shown along with the results in the shift-reflect (SR) symmetric subspace (blue). The full space attractor displays an increasing trend in $\left\|\hat{\alpha}_{x x}\right\|_{2}$ from Newtonian to $\mathrm{Wi}=8$ followed by a decrease up to $\mathrm{Wi}=11$ and an increase beyond that. The instantaneous dynamics of $\left\|\hat{\alpha}_{x x}\right\|_{2}$ go from 2-torus dynamics up to $\mathrm{Wi}=2$ to mild chaos at $\mathrm{Wi}=4$. As $\mathrm{Wi}$ further increases, the dynamics get more complicated and reach a peak in intermittency around $\mathrm{Wi}=11$. Here, intermittent bursts characterized by a sharp temporary increase in the instantaneous $\left\|\hat{\alpha}_{x x}\right\|_{2}$ can be seen as a persistent phenomena.

Many of the observations described in the full space carry forward to the SR subspace up to $\mathrm{Wi}=10$. The same nonmonotonic trend in $\left\|\hat{\alpha}_{x x}\right\|_{2}$ is accompanied by a temporal modulation around $\mathrm{Wi}=4$. In other words, the full space attractor can be seen as a modification of the attractor in the $\mathrm{SR}$ subspace. Beyond $\mathrm{Wi}=10$, the dynamics in the SR space alternate between two metastable states (indicated by vertical dotted lines) before settling on the stable symmetric attractor at $\mathrm{Wi}=$ 13. We will expand on this observation in Sec. III C. 


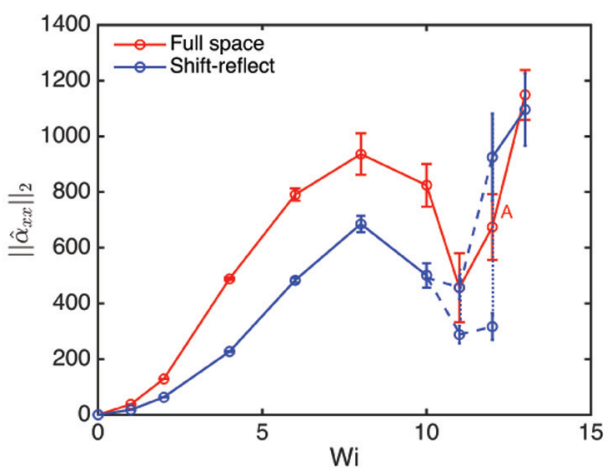

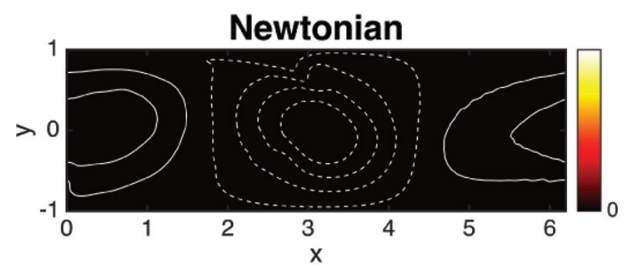

(b)

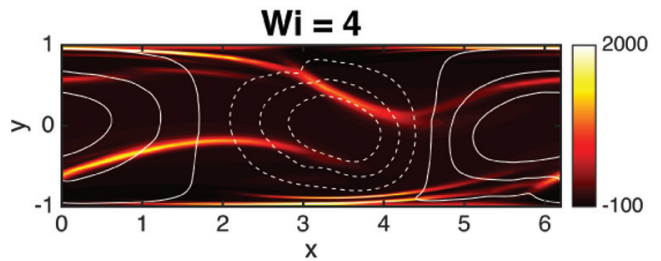

(d)

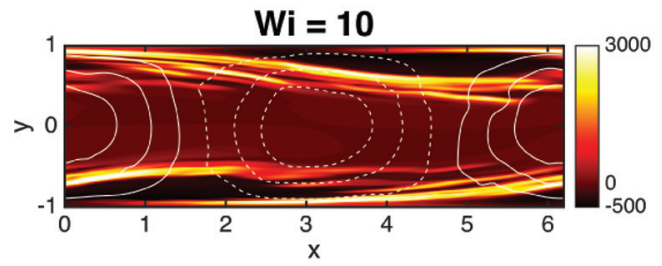

(f)

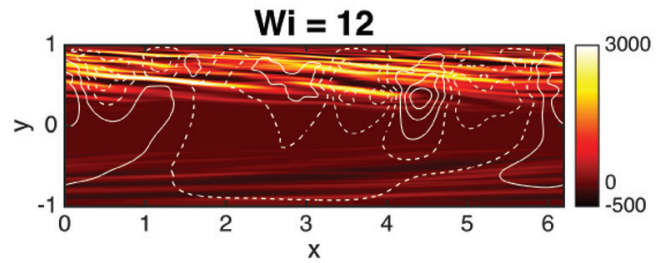

(h)

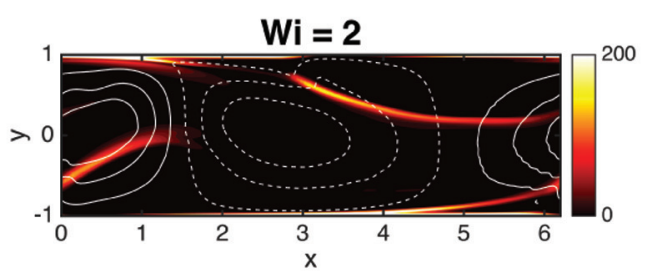

(c)

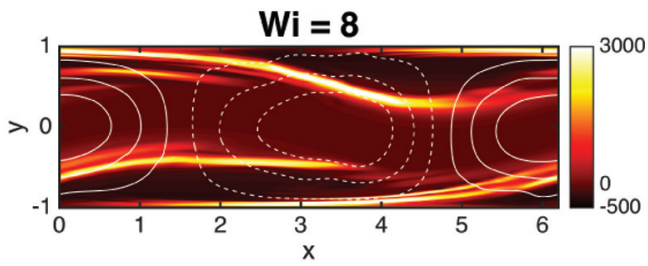

(e)

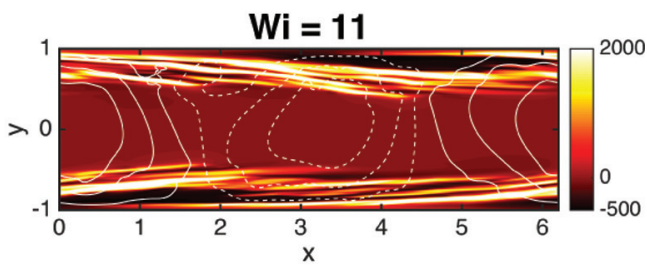

(g)

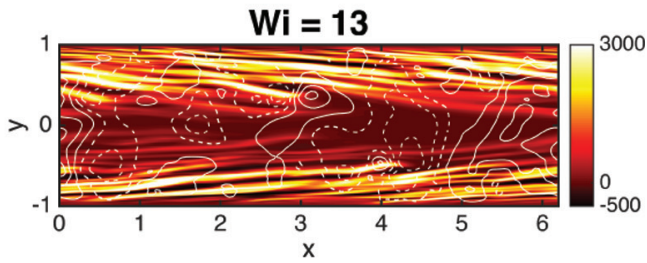

(i)

FIG. 3. (a) $\left\|\hat{\alpha}_{x x}\right\|_{2}$ vs $\mathrm{Wi}$ at $\mathrm{Re}=10000$ for simulations in the full space and the shift-reflect subspace. Here, "A" indicates asymmetry in the attractor in the full space. The upper and lower blue symbols at Wi $=11$ and 12 indicate averages over the two metastable states intermittently visited by the dynamics at these Wi values. (b)-(i) are snapshots of the fluctuation structure at the indicated Wi. 


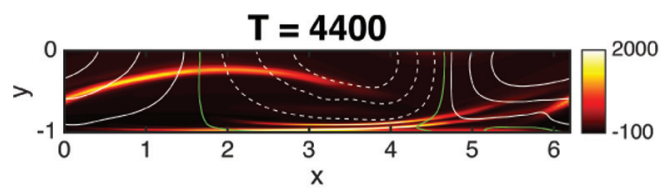

(a)

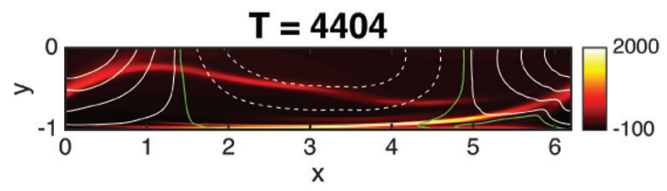

(c)

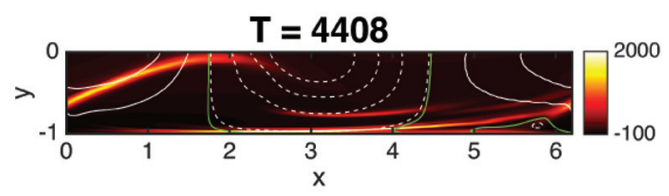

(e)

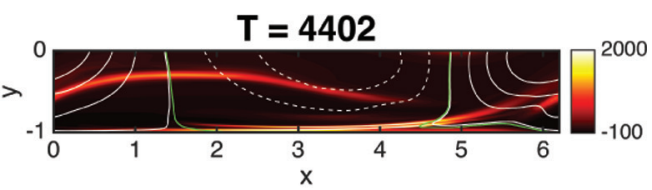

(b)

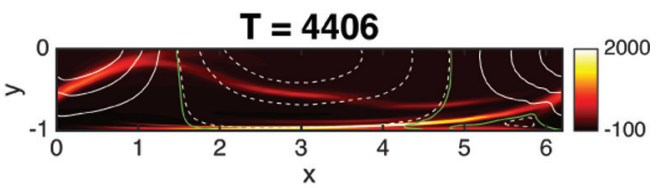

(d)

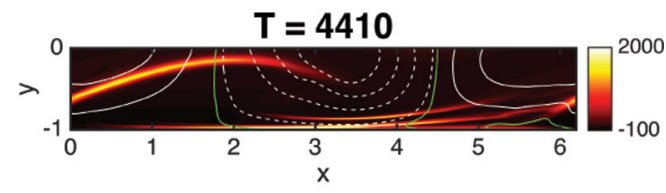

(f)

FIG. 4. (a)-(f) Snapshots of the fluctuation structure at $\mathrm{Re}=10000, \mathrm{Wi}=4$. Snapshots are taken from $t=4400$ to 4410 every 2 time units, respectively. Green lines correspond to $\hat{v}=0$, i.e., where wall normal velocity changes sign. Bottom half of the domain shown.

To get a sense of what underlies the dynamics, we now turn to the full-space flow structures at various values of Wi. Snapshots of these structures are shown in Figs. 3(b)-3(i). The Newtonian attractor [Fig. 3(b)] displays the usual wall normal velocity contour lines across the channel height. At Wi $=2$ [Fig. 3(c)], the attractor develops a sheet of polymer stretch that starts near the wall and arches closer to the center line. This is typical of the NNTSA structure described in the Introduction and can be attributed to the Kelvin cat's eye critical layer kinematics of the attractor. Going from $\mathrm{Wi}=2$ to 4 , the attractor starts to display near-wall multilayered sheets, as seen between $x \approx 3$ and $x \approx 5$ in Fig. 3(d). This effect gets more pronounced as we move along the branch, with $\mathrm{Wi}=11$ intermittently displaying strong multilayered sheets [Fig. 3(g)] similar to those seen at EIT at Wi $=$ 13 [Fig. 3(i)]. Interestingly, Wi $=12$ displays strong multilayered sheets on one-half of the channel with no particular preference towards either half. (By symmetry, for every state with fluctuations localized near the top, there is a dynamically equivalent state localized near the bottom. Asymmetric states similar to this have been observed in 2D Newtonian channel flow [39].) The marked structural transition beyond $\mathrm{Wi}=11$ coincides with an increasing trend in $\left\|\hat{\alpha}_{x x}\right\|_{2}$ in Fig. 3(a) and gives rise to the EIT branch beyond $\mathrm{Wi}=11$. We now describe the process through which this branch makes the structural transition from a single sheet at low Wi to the multilayered sheets seen at EIT.

To elaborate on this sheet shedding process, we focus on the attractor at $\mathrm{Re}=10000$, Wi $=4$, i.e., close to where this process first comes into existence. Figures 4(a) to 4(f) cover one cycle of the process and correspond to $t=4400$ to 4410 taken every 2 time units, respectively. The following description focuses on the bottom half of the channel with the same process taking place in the top half.

As the name suggests, this process involves the shedding of sheets of polymer stretch from the primary TS structure, giving rise to multilayered sheets. The onset of this process involves the arched portion of the TS structure seen between $x=0$ and $x \approx 4$. At the instant shown in (a), this arched section goes from a region of positive wall normal velocity to negative. The locations where the wall normal velocity changes sign are shown using green lines as seen near $x \approx 1.5$. Going from instant (a) to (c), this arch gets weaker and stretches out due to the wall normal velocity pulling the arch towards the center line for $x \lesssim 1.5$ and towards the wall beyond this region. Eventually, a piece 
of this arch breaks off, i.e., it is "shed" as shown in (d) near the location where the wall normal velocity changes sign.

The next stage of the process has to do with these fragments that are shed, which get convected downstream faster than the primary TS structure. This can be seen in (e). This difference has to do with the fact that the velocity of the primary TS structure is dictated by the mean velocity at the critical layer while these fragments are closer to the center line, where the streamwise velocity is higher. As these fragments are convected, they are simultaneously sheared, giving rise to the near-wall multilayered sheet seen in (f) between $x \approx 3$ and $x \approx 6$. The instant (f) is similar to (a), and the process repeats. The time scale of the process is dictated by the dominant time scale of the attractor with TS critical layer mechanisms at its core.

This process gets more pronounced as we move along this branch, and the statistical intermittency described earlier corresponds to a structural intermittency due to the sheet shedding process excited by viscoelasticity. To show this, Fig. 5 depicts the structural intermittency observed at $\mathrm{Wi}=11$. Snapshots shown are taken every $10 \mathrm{TU}$-the time scale at which we described the sheet shedding process at $\mathrm{Wi}=4$. The attractor goes from displaying a single sheet of polymer stretch as seen in (a) to strong multilayered sheets at the instant (j) and back. The observed steps of shedding-convecting-shearing now happen in a fashion that eventually gives rise to multilayered sheets. These near-wall sheets observed during this intermittent process start to resemble those observed at EIT as described earlier. These multilayered sheets take over the dynamics at $\mathrm{Wi}=12$, turning into EIT at $\mathrm{Wi}=13$.

To build on the evidence linking the TS attractor (up to Wi = 11) and EIT (beyond 11), Fig. 6 shows the power spectral density of wall normal velocity at $y= \pm 0.8$, with the following observations holding true at other $y$ locations. At Wi $=11$ (blue line), the spectrum is mainly composed of the fundamental TS frequency (red-dashed line) and its two higher harmonics (black-dashed line). This is unsurprising, as TS-like flow structures characterized by wall-normal velocity contour lines spanning the channel are a dominant feature of the attractor (evidenced by Fig. 5). Interestingly, the fundamental TS frequency (red-dashed vertical line) remains a prominent feature of the spectrum even at $\mathrm{Wi}=13$ (brown line) in spite of the obvious structural differences with $\mathrm{Wi}=11$. This points at TS-like mechanisms dictating the dynamics even at EIT.

Looking at other statistics, Fig. 7 shows the evolution of mean profiles of streamwise velocity [Fig. 7(a)], Reynolds shear stress [Fig. 7(b)], and polymer shear stress [Fig. 7(c)] going from the Newtonian attractor to EIT. On increasing Wi, the mean velocity profiles get increasingly uplifted from Newtonian (red line) to $\mathrm{Wi}=11$ (purple line) with the profile at $\mathrm{Wi}=4$ (blue line) very similar to the Newtonian one. EIT at $\mathrm{Wi}=13$ (green line) displays a profile in between Newtonian and $\mathrm{Wi}=11$. Turning to the Reynolds shear stress profiles, the Newtonian profile (red line) displays a near-wall peak corresponding to the critical layer of the TS wave. This points to a prominent role for critical layer behavior in Reynolds shear stress production. Moving along the branch, the attractors continue to display this near-wall peak with a monotonic decrease in magnitude until $\mathrm{Wi}=13$, where the Reynolds shear stress becomes negligible. Past studies of EIT have reported similar observations [16,18,21]. While Reynolds shear stress dominates over polymer shear stress at $\mathrm{Wi}=4$, the roles are completely reversed at EIT, with polymer shear stress being the main source of turbulence generation. At $\mathrm{Wi}=11$, the above quantities are of similar magnitude, thus indicating roles of equal importance in self-sustenance. Further, the peak polymer shear stress at EIT loosely aligns with the peaks at lower Wi. This hints at mechanisms originating from the TS attractor underlying the main source of turbulence production at EIT. Taken together, these results provide direct indications of a connection between the TS attractor and EIT.

\section{Intermittent regime: Analogy with relaxation oscillations}

In the range $10 \lesssim \mathrm{Wi} \lesssim 13$, the amplitude of $\left\|\hat{\alpha}_{x x}\right\|_{2}$ increases sharply and its temporal fluctuations are large. At the beginning of this regime, the flow remains structurally similar to the TS wave, but with substantial sheet shedding [Fig. 3(f), Wi = 10], while at the end is found the full-blown EIT structure, with sheets occupying most of the channel [Fig. 3(i)]. To gain some understanding 


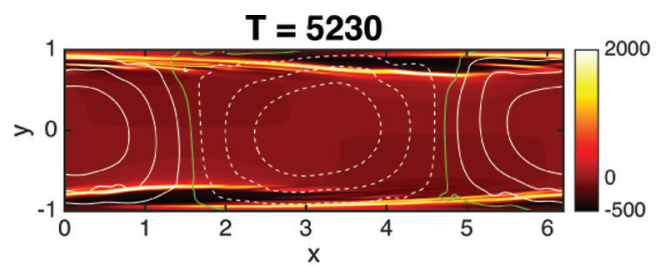

(a)

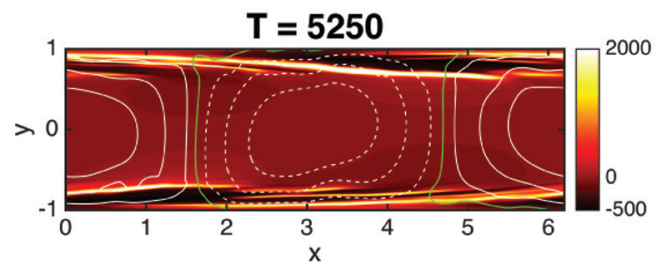

(c)

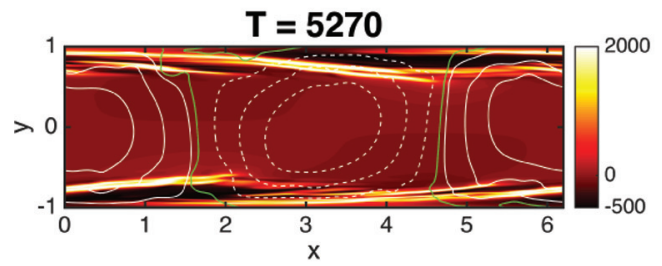

(e)

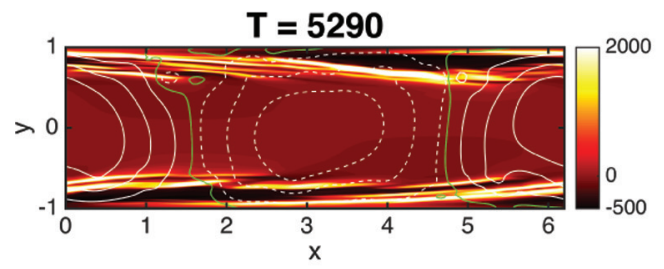

(g)

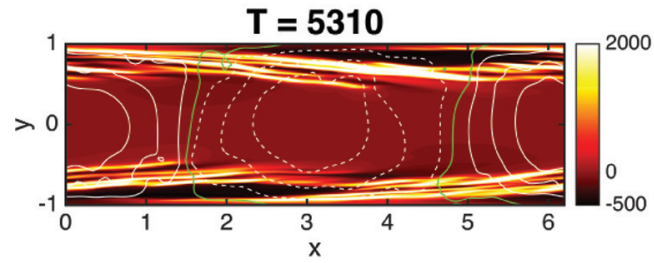

(i)

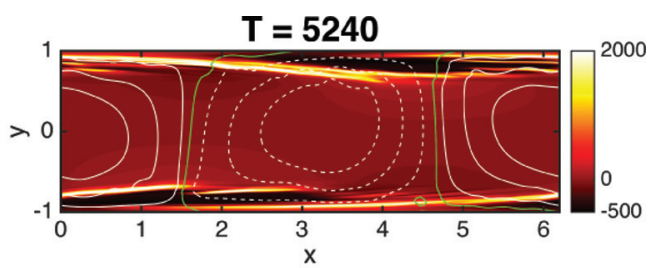

(b)

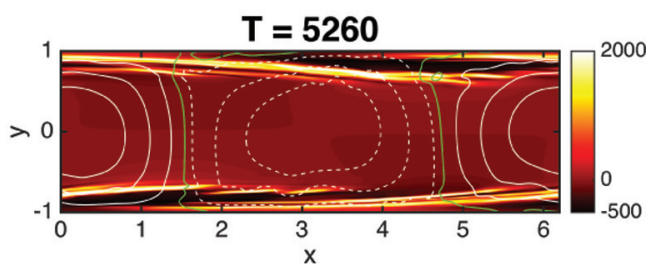

(d)

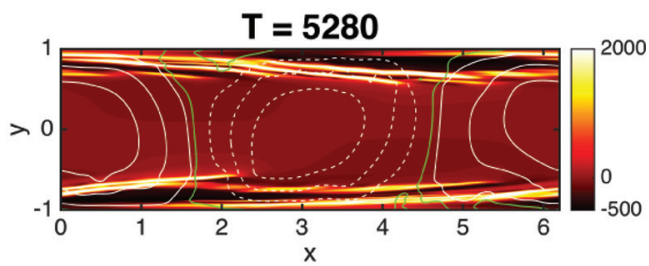

(f)

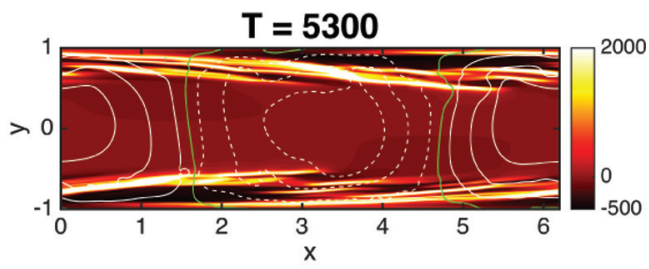

(h)

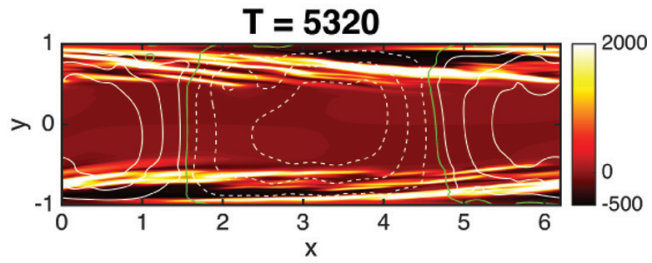

(j)

FIG. 5. (a)-(j) Snapshots of the fluctuation structure at Wi $=11$. Snapshots are taken from $t=5230$ to 5320 every 10 time units, respectively. Same format as Fig. 4.

of this transition, we focus on the dynamics in the shift-reflect subspace. With this restriction, the intermittency and basic structural features of the full space simulations are found, while the symmetry breaking that leads to the degenerate, strongly asymmetric structures like that in Fig. 3(h) will not complicate matters.

Figure 8(a) shows the time evolution of the instantaneous value of $\left\|\hat{\alpha}_{x x}\right\|_{2}$ from simulations in the SR subspace at $\mathrm{Wi}=11,12$, and 13 . While there are substantial fluctuations in the flows at 11 and 13 , the behavior at $\mathrm{Wi}=12$ is quite distinct, displaying strong intermittency between two 


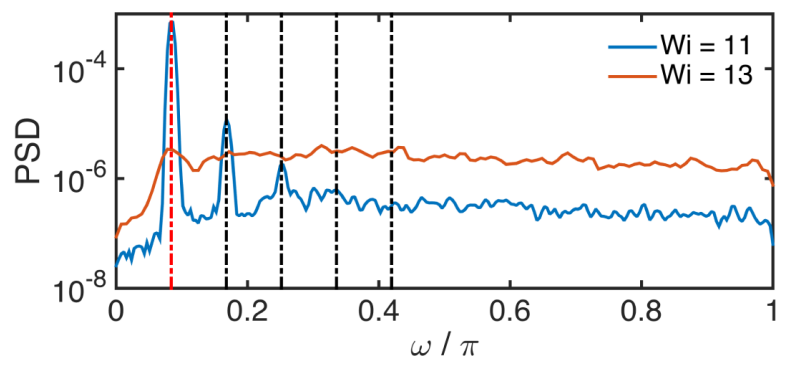

FIG. 6. Power spectral density (PSD) of wall normal velocity at $y= \pm 0.8$ at the indicated Wi.

metastable states. A snapshot from the lower of these states [Fig. 8(b)] closely resembles a TS wave, while one from the upper [Fig. 8(b)] looks like EIT. There is no hysteresis in this scenario: if we use initial conditions at higher $\mathrm{Wi}$ to generate trajectories at lower $\mathrm{Wi}$, we get the same behavior as when we use initial conditions at lower Wi to generate trajectories at higher Wi.

The destabilization of one attractor (TS here) leading to a regime of intermittency followed by the appearance of another attractor with substantially different amplitude is typical of a dynamical system with multiple time scales with a pleated slow manifold and the possibility of rapid jumps

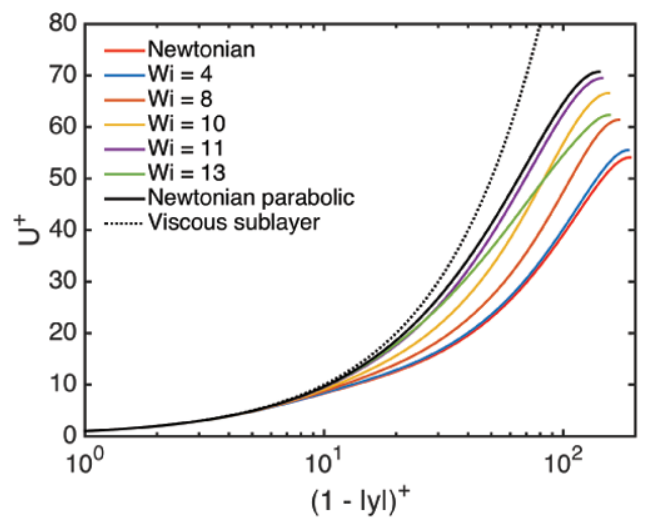

(a)

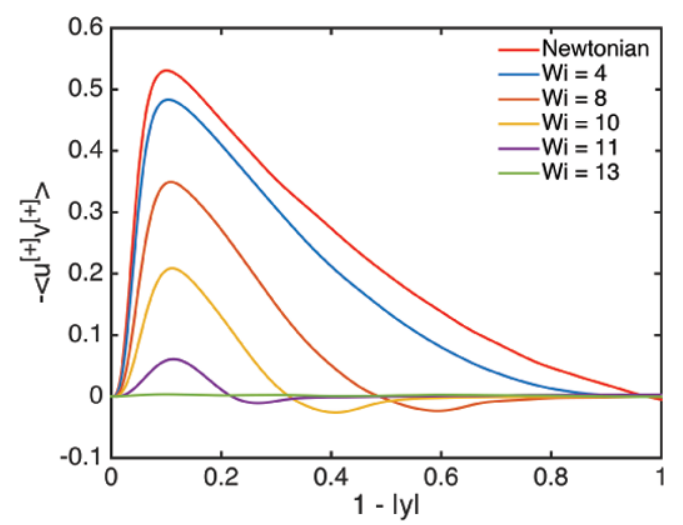

(b)

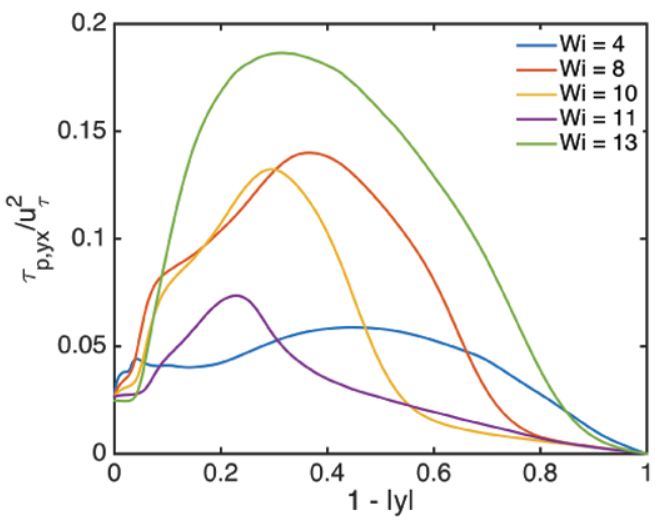

(c)

FIG. 7. (a) Mean streamwise velocity profiles, (b) Reynolds shear stress profiles, and (c) polymer shear stress profiles of full space solutions. All profiles are shown as a function of distance from the wall $1-|y|$. 


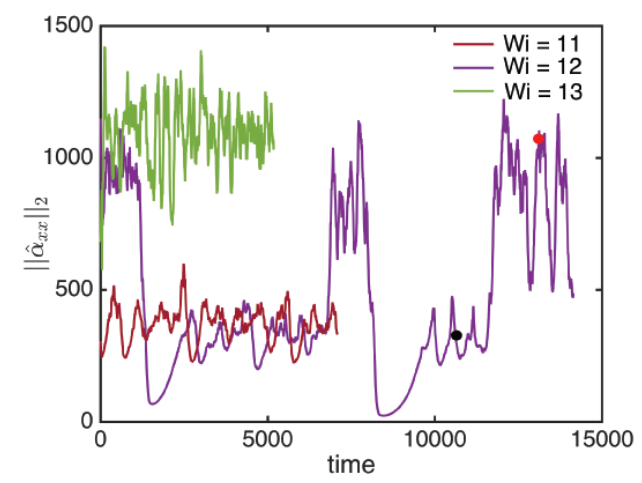

(a)

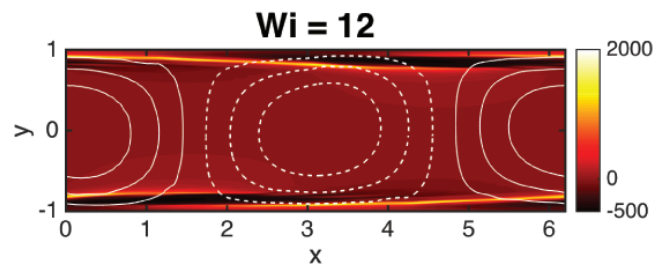

(b)

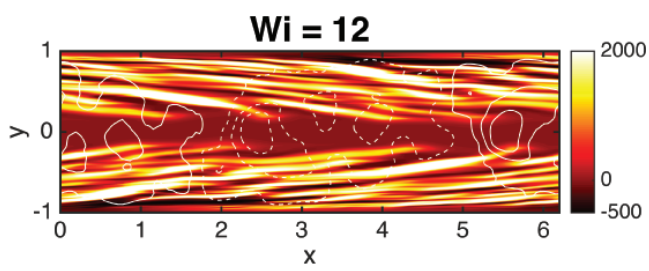

(c)

FIG. 8. (a) Instantaneous $\left\|\hat{\alpha}_{x x}\right\|_{2}$ vs time at $\mathrm{Re}=10000$ in the shift-reflect symmetric subspace. Panels (b) and (c) are snapshots of the fluctuation structure of the TS (instant indicated by the black dot) and EIT (red dot) metastable states, respectively.

between different, locally attracting, pleats [40]. In particular, we draw an analogy with the following van der Pol-type system:

$$
\begin{gathered}
\dot{x}=\frac{1}{\epsilon}\left[y-\left(x^{3} / 3-x\right)\right], \\
\dot{y}=a-x,
\end{gathered}
$$

Here, $a$ is the control parameter of the system and $\epsilon$ determines the ratio of time scales between the $x$ and $y$ dynamics. The classical van der Pol system is recovered when $a=0$. At each value of $a$, there is a unique steady state $(x, y)=\left(a, a^{3} / 3-a\right)$. As $\epsilon \rightarrow 0$, this state is stable when $a<-1$ and $a>1$, while, in $-1<a<1$, there is a stable limit cycle-a relaxation oscillation that jumps rapidly between positive and negative values of $x$. The bifurcations are all supercritical so as in the above scenario there is no hysteresis. Figure 9 shows the $x$ dynamics of the system for $\epsilon=0.01$ at different values of $a$. For $a=-1.1$ (maroon line) and $a=1.1$ (green line), initial conditions settle on the stable steady state $x=-1.1$ and $x=1.1$, respectively. For $a=0$ (purple line), the system displays relaxation oscillations. The observations presented in this section, taken in consideration with evidence of a link between TS and EIT, are entirely consistent with a high-dimensional version of such relaxation oscillations between TS and EIT metastable states at $\mathrm{Wi}=12$, which give rise to stable EIT at higher Wi.

\section{Robustness in large spatial domains and very high polymer extensibility}

Finally, we present results in other parameter regimes to portray the robustness of our observations. For each of the below scenarios, we look at snapshots of the attractor for different levels of viscoelasticity.

First, we consider results at $\mathrm{Re}=10000$ in a large domain of size $L_{x}=31$, i.e., five times the domain size used above. Snapshots of the attractor are shown in Fig. 10. At Wi $=6$, the attractor 


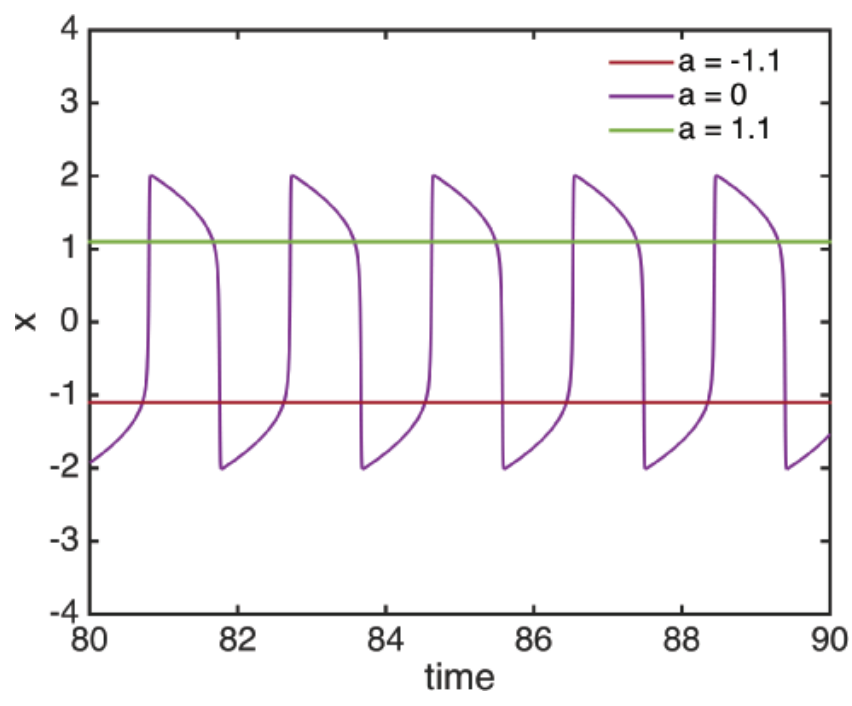

FIG. 9. Dynamics of $x$ in the van der Pol-type system described by Eqs. (6) and (7) for $\epsilon=0.01$ at the indicated values of control parameter $a$.

displays a structure corresponding to five spatial periods of the TS attractor in $L_{x}=6.2$, with nearwall sheets and wall normal velocity across the channel. Further, we can see clear evidence of the sheet shedding process at play. Here, different parts of the domain can be at different stages of the process: i.e., there is spatiotemporal intermittency. For example, we see multilayered sheets near $x=10$ and sheet shedding near $x=25$. At Wi $=13$, we see EIT with strong multilayered sheets of polymer stretch as we did for $L_{x}=6.2$.

Now we consider the scenario at very high extensibility, $b=100000$, with other parameters remaining the same. Figure 11 depicts snapshots of the attractor for this case. At Wi $=4$, the attractor corresponds to the TS attractor in this parameter regime and displays the same characteristics as the attractor at lower extensibility. Further, the attractor continues to exhibit the sheet shedding process that leads to multilayered sheets of polymer stretch. This can be seen near $x=4$. On increasing Wi, the TS attractor loses stability before giving rise to stable EIT. At $\mathrm{Wi}=6$, the dynamics alternate between TS and EIT metastable states as shown in Figs. 11(b) and 11(c), respectively. This EIT metastable state can sometimes be asymmetric and thus in agreement with the observations at $\mathrm{Wi}=12, b=6400$. These results are consistent with the relaxation oscillation scenario above with the bifurcation happening around $\mathrm{Wi} \approx 6$ at this value of extensibility. At $\mathrm{Wi}=13$, we observe EIT.

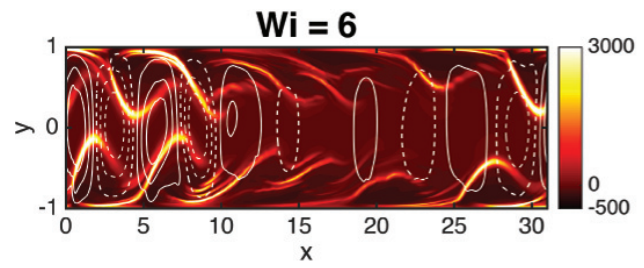

(a)

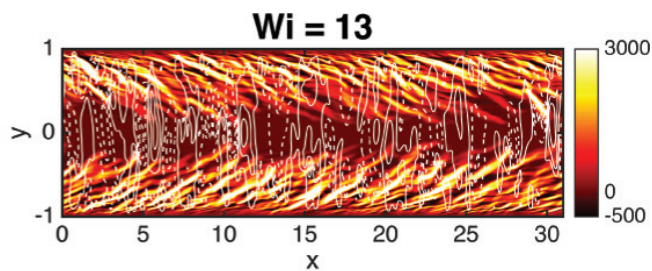

(b)

FIG. 10. Snapshots of the attractor at $\mathrm{Re}=10000$ in a box of size $L_{x}=31$ at the indicated Wi. Contour plots follow the same format as previous figures. Note the compressed scale in $x$. 


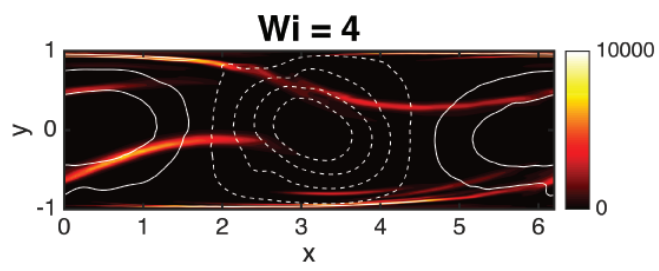

(a)

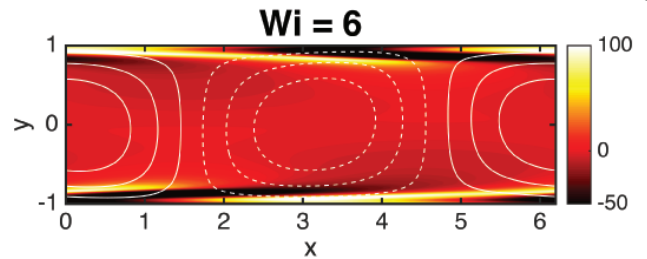

(b)

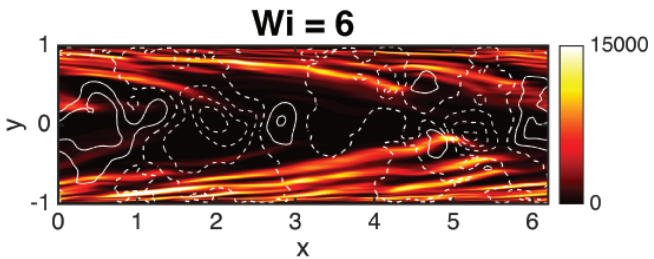

(c)

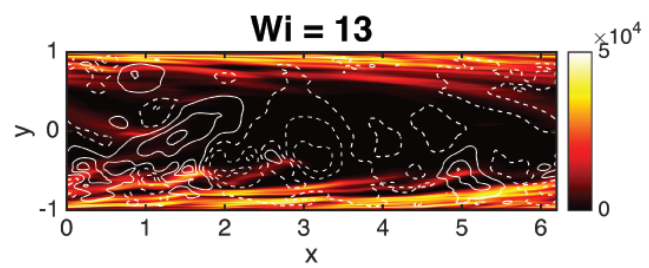

(d)

FIG. 11. Snapshots of the attractor at $\mathrm{Re}=10000$ with $b=100000$ at various Wi. At Wi $=6$, the dynamics are strongly intermittent; (b) and (c), respectively, are snapshots from the low-amplitude (TS) and large amplitude (EIT) intervals. Format is the same as previous plots.

\section{E. Discussion: Flow geometry and dimensionality}

As with our prior study, Ref. [15], the present work is limited to two-dimensional flow in a plane channel. Given these limitations, it is important to consider how they may be viewed in the broader context of turbulent drag reduction, first with regard to how they may relate to the pipe flow geometry and then in the full context of three-dimensional turbulence. These issues were discussed at some length in Ref. [15], so here we summarize that discussion and describe some further issues in connection to very recent literature.

As noted in the Introduction, elastoinertial turbulence with very similar features has been observed in channel, pipe, and plane Couette flows. A natural question, then, is to what extent the above channel flow results are relevant to the pipe flow case. While it is true of course that there is no linear instability of Newtonian pipe or plane Couette flows, there are nevertheless "wall modes" with critical layer structure related to that of the TS mode [36]. Additionally, critical layers are a strong source of linear amplification in those flows, as they are in channel flow [26]. Furthermore, the absence of a Newtonian mechanism for nonlinear sustainment of Tollmien-Schlichting-like traveling waves does not imply the absence of a viscoelastic mechanism. Indeed, Shekar et al. [15] showed the existence of just such a mechanism. Furthermore, as noted in the Introduction, pipe flow [24] and plane Couette flow [28] simulations at EIT display essentially two-dimensional velocity fluctuations localized near the wall that are similar to those reported in channel flow, and resolvent analysis of viscoelastic pipe flow demonstrates strong linear amplification of modes with near-wall critical-layer stress fluctuations [27].

At the same time, in more strongly viscoelastic regimes, Garg et al. [41] and Khalid et al. [42] have found a center mode instability for pipe flow and channel flow, respectively, in the Oldroyd-B limit. To put these results into context, for $\mathrm{Re}=10000$, this instability comes into 
existence for $\mathrm{Wi} \gtrsim 500$ for $\beta=0.97$ and $\mathrm{Wi} \gtrsim 200$ for $\beta=0.9$ in either geometry. Reference [43] numerically converged nonlinear "arrowhead" structures originating subcritically from this instability in channels at very low $\operatorname{Re} \sim O(100)$ and $\mathrm{Wi} \sim O(10)$. Similar structures were observed in 2D simulations of EIT by Dubief et al. [44] at very high viscoelasticity with Wi $\sim O(100)$. Choueiri et al. [25] also note the appearance of "chevron" shaped structures resembling the unstable center mode in pipe flow at very low $\operatorname{Re} \sim O(100)$ before being taken over by near-wall modes on increasing Re. These results open up the possibility that other states unrelated to the nonlinear excitation of a wall mode may also play a role at EIT in both channels and pipes, especially at very low Re and high Wi. Nevertheless, the present work demonstrates a direct connection between a Newtonian wall mode (the TS mode) and two-dimensional EIT structures.

We now turn to the topic of how the present results are related to the fully three-dimensional context of near-wall turbulence. Newtonian turbulence is, of course, strongly three dimensional, with the dominant near-wall structure comprised of coherent wavy streamwise vortices that to some extent can be modeled by so-called exact coherent states (ECS) [14]. In particular, Li et al. [13] studied the bifurcation scenario for a particular family of channel flow ECS [45] in a parameter regime close to that considered here. At $\mathrm{Re}=1500$, this ECS family is sufficiently weakened by viscoelasticity to lose existence at $\mathrm{Wi} \approx 16$, somewhat above the value $\mathrm{Wi} \approx 10$ beyond which Newtonian turbulence cannot self-sustain in the DNS study of [18]. (This discrepancy is consistent with what we know about transition in the Newtonian case: channel flow turbulence is self-sustaining above $\operatorname{Re} \approx 1000$, while ECS can exist in that case down to $\operatorname{Re} \approx 660$ [46].) Extrapolating slightly from the results of $\mathrm{Li}$ et al. [13], one can estimate that, at $\mathrm{Re}=10000$, this ECS family exists at least up to $\mathrm{Wi}=25$. Based on the results above, we might expect that, when $\mathrm{Wi} \gtrsim 13$, a mix of EIT-like and quasistreamwise structures might coexist, while, once Wi $>25$, two-dimensional EIT would dominate. Consistent with this analysis, our preliminary work [47] as well as a number of other recent studies have reported coexistence of EIT-like sheets and 3D quasistreamwise structures $[17,28,29,48-50]$. How the 2D and 3D structures interact is an important topic for future work.

\section{CONCLUSION}

This study describes the evolution of the Tollmien-Schlichting (TS) attractor in Re-Wi parameter space and its relationship to elastoinertial turbulence (EIT), using simulations of 2D channel flow of a very dilute polymer solution. At $\mathrm{Re}=3000$, there is a solution branch with TS-wave structure but which is not connected to the Newtonian solution branch. At fixed Weissenberg number, Wi, and increasing Reynolds number, Re, from 3000 to 10 000, this attractor goes from displaying a striation of weak polymer stretch localized at the critical layer as described in Shekar et al. [15] to an extended sheet of very large polymer stretch. We show that this transition is directly tied to the strength of the TS critical layer fluctuations and can be attributed to a coil-stretch transition when the local Wi at the hyperbolic stagnation point of the Kelvin cat's eye structure of the TS wave exceeds $\frac{1}{2}$. At $\operatorname{Re}=10000$, unlike 3000, the Newtonian TS attractor evolves continuously into EIT as Wi is increased from zero to about 13-the two flows are directly connected in parameter space. We describe how the structure of the flow and stress fields changes with Wi, highlighting in particular a sheet-shedding process by which the individual sheets associated with the critical layer structure break up to form the layered multisheet structure characteristic of EIT. Furthermore, in an intermediate parameter regime a state of highly intermittent dynamics are observed: the flow behavior alternates between a state with sheetlike stress fluctuations localized near the wall-the TS structure-to one with such structures present across the channel, except near the center line-EIT. To understand this regime, we draw analogies with relaxation oscillator dynamics.

The linear instability to Tollmien-Schlichting waves does not arise for pipe or plane Couette flow, so the scenario described here does not directly apply to those geometries. On the other hand, in these geometries elastoinertial turbulence with very similar features does arise in simulations in 
the same general parameter regime: namely, fluctuations localized in a layer near the wall, with a sheetlike stress structure and little to no activity in the center of the flow [24,28]. Furthermore, while linearly stable, wall modes analogous to the TS wave do exist in these other geometries and may be subject to nonlinear critical layer excitation and subsequent evolution into EIT, just as the TS mode is in the channel flow case.

\section{ACKNOWLEDGMENTS}

This work was supported by Grants No. (UW) NSF CBET-1510291, No. AFOSR FA9550-18-10174, and No. ONR N00014-18-1-2865, and Grant No. (Caltech) ONR N00014-17-1-3022.

[1] B. A. Toms, Some observations on the flow of linear polymer solutions through straight tubes at large Reynolds numbers, in Proceedings of the 1st International Congress on Rheology (North-Holland, Amsterdam, 1949), Vol. 2, pp. 135-141.

[2] B. A. Toms, On the early experiments on drag reduction by polymers, Phys. Fluids 20, S3 (1977).

[3] J. K. Fink, Petroleum Engineer's Guide to Oil Field Chemicals and Fluids (Gulf Professional Publishing, Houston, TX, 2012).

[4] E. Burger, W. Munk, and H. Wahl, Flow increase in the trans Alaska pipeline through use of a polymeric drag-reducing additive, J. Pet. Technol. 34, 377 (1982).

[5] G. E. King, Hydraulic fracturing 101: What every representative, environmentalist, regulator, reporter, investor, university researcher, neighbor and engineer should know about estimating frac risk and improving frac performance in unconventional gas and oil wells, in SPE Hydraulic Fracturing Technology Conference (Society of Petroleum Engineers, The Woodlands, TX, 2012).

[6] K. Kim, C.-F. Li, R. Sureshkumar, S. Balachandar, and R. J. Adrian, Effects of polymer stresses on eddy structures in drag-reduced turbulent channel flow, J. Fluid Mech. 584, 281 (2007).

[7] C. M. White and M. G. Mungal, Mechanics and prediction of turbulent drag reduction with polymer additives, Annu. Rev. Fluid Mech. 40, 235 (2008).

[8] P. Stone, F. Waleffe, and M. D. Graham, Toward a Structural Understanding of Turbulent Drag Reduction: Nonlinear Coherent States in Viscoelastic Shear Flows, Phys. Rev. Lett. 89, 208301 (2002).

[9] P. Stone and M. D. Graham, Polymer dynamics in a model of the turbulent buffer layer, Phys. Fluids 15, 1247 (2003).

[10] P. A. Stone, A. Roy, R. G. Larson, F. Waleffe, and M. D. Graham, Polymer drag reduction in exact coherent structures of plane shear flow, Phys. Fluids 16, 3470 (2004).

[11] W. Li, P. A. Stone, and M. D. Graham, Viscoelastic nonlinear traveling waves and drag reduction in plane Poiseuille flow, Proceedings of the IUTAM Symposium on Laminar-Turbulent Transition and Finite Amplitute Solutions, Fluid Mechanics and its Applications Vol. 77 (Springer, Dordrecht, 2005), pp. 289312.

[12] W. Li, L. Xi, and M. D. Graham, Nonlinear travelling waves as a framework for understanding turbulent drag reduction, J. Fluid Mech. 565, 353 (2006).

[13] W. Li and M. D. Graham, Polymer induced drag reduction in exact coherent structures of plane Poiseuille flow, Phys. Fluids 19, 083101 (2007).

[14] M. D. Graham and D. Floryan, Exact coherent states and the nonlinear dynamics of wall-bounded turbulent flows, Annu. Rev. Fluid. Mech. 53, 227 (2021).

[15] A. Shekar, R. M. McMullen, B. J. McKeon, and M. D. Graham, Self-sustained elastoinertial TollmienSchlichting waves, J. Fluid Mech. 897, A3 (2020).

[16] D. Samanta, Y. Dubief, M. Holzner, C. Schäfer, A. N. Morozov, C. Wagner, and B. Hof, Elasto-inertial turbulence, Proc. Natl. Acad. Sci. USA 110, 10557 (2013).

[17] G. H. Choueiri, J. M. Lopez, and B. Hof, Exceeding the Asymptotic Limit of Polymer Drag Reduction, Phys. Rev. Lett. 120, 124501 (2018). 
[18] A. Shekar, R. M. McMullen, S.-N. Wang, B. J. McKeon, and M. D. Graham, Critical-Layer Structures and Mechanisms in Elastoinertial Turbulence, Phys. Rev. Lett. 122, 124503 (2019).

[19] V. E. Terrapon, Y. Dubief, and J. Soria, Dynamics of elasto-inertial turbulence in flows with polymer additives, in Eighth International Symposium on Turbulence and Shear Flow Phenomena (Begell House Inc., Redding, CT, 2013).

[20] V. E. Terrapon, Y. Dubief, and J. Soria, On the role of pressure in elasto-inertial turbulence, J. Turbul. 16, 26 (2014).

[21] S. Sid, V. E. Terrapon, and Y. Dubief, Two-dimensional dynamics of elasto-inertial turbulence and its role in polymer drag reduction, Phys. Rev. Fluids 3, 011301 (2018).

[22] S. J. Haward, J. Page, T. A. Zaki, and A. Q. Shen, "Phase diagram" for viscoelastic Poiseuille flow over a wavy surface, Phys. Fluids 30, 113101 (2018).

[23] S. J. Haward, J. Page, T. A. Zaki, and A. Q. Shen, Inertioelastic Poiseuille flow over a wavy surface, Phys. Rev. Fluids 3, 091302 (2018).

[24] J. M. Lopez, G. H. Choueiri, and B. Hof, Dynamics of viscoelastic pipe flow at low Reynolds numbers in the maximum drag reduction limit, J. Fluid Mech. 874, 699 (2019).

[25] G. H. Choueiri, J. M. Lopez, A. Varshney, S. Sankar, and B. Hof, Experimental observation of the origin and structure of elasto-inertial turbulence, arXiv:2103.00023.

[26] B. J. McKeon and A. S. Sharma, A critical-layer framework for turbulent pipe flow, J. Fluid Mech. 658, 336 (2010).

[27] M. Zhang, Energy growth in subcritical viscoelastic pipe flows, J. Non-Newtonian Fluid Mech. 294, 104581 (2021).

[28] A. Pereira, R. L. Thompson, and G. Mompean, Beyond the maximum drag reduction asymptote: the pseudo-laminar state, arXiv:1911.00439.

[29] L. Zhu et al., Nonasymptotic elastoinertial turbulence for asymptotic drag reduction, Phys. Rev. Fluids 6 , 014601 (2021).

[30] J. Jiménez, Transition to turbulence in two-dimensional Poiseuille flow, J. Fluid Mech. 218, 265 (1990).

[31] A. Kurganov and E. Tadmor, New high-resolution central schemes for nonlinear conservation laws and convection-diffusion equations, J. Comput. Phys. 160, 241 (2000).

[32] T. Vaithianathan, A. Robert, J. G. Brasseur, and L. R. Collins, An improved algorithm for simulating three-dimensional, viscoelastic turbulence, J. Non-Newtonian Fluid Mech. 140, 3 (2006).

[33] V. Dallas, J. Vassilicos, and G. Hewitt, Strong polymer-turbulence interactions in viscoelastic turbulent channel flow, Phys. Rev. E 82, 066303 (2010).

[34] A. Kushwaha, Near-wall turbulence and utilisation of the nonlinear dynamics towards control of turbulent flows, Ph.D. thesis, University of Wisconsin-Madison, 2017.

[35] S.-N. Wang, Nonlinear dynamics of viscoelastic turbulence in transitional channel flow, Ph.D. thesis, University of Wisconsin-Madison, 2017.

[36] P. G. Drazin and W. H. Reid, Hydrodynamic Stability, 2nd ed., Cambridge Mathematical Libraries (Cambridge University Press, Cambridge, UK, 2004).

[37] P. De Gennes, Coil-stretch transition of dilute flexible polymers under ultrahigh velocity gradients, J. Chem. Phys. 60, 5030 (1974).

[38] M. D. Graham, Microhydrodynamics, Brownian Motion, and Complex Fluids, Cambridge Texts in Applied Mathematics (Cambridge University Press, Cambridge, UK, 2018).

[39] V. K. Markeviciute and R. R. Kerswell, Degeneracy of turbulent states in two-dimensional channel flow, J. Fluid Mech. 917, A57 (2021).

[40] J. Guckenheimer, Bifurcations of relaxation oscillations, in Normal Forms, Bifurcations and Finiteness Problems in Differential Equations (World Scientific, Singapore, 2004), pp. 295-316.

[41] P. Garg, I. Chaudhary, M. Khalid, V. Shankar, and G. Subramanian, Viscoelastic Pipe Flow is Linearly Unstable, Phys. Rev. Lett. 121, 024502 (2018).

[42] M. Khalid, I. Chaudhary, P. Garg, V. Shankar, and G. Subramanian, The centre-mode instability of viscoelastic plane Poiseuille flow, J. Fluid Mech. 915, A43 (2021).

[43] J. Page, Y. Dubief, and R. R. Kerswell, Exact Traveling Wave Solutions in Viscoelastic Channel Flow, Phys. Rev. Lett. 125, 154501 (2020). 
[44] Y. Dubief, J. Page, R. R. Kerswell, V. E. Terrapon, and V. Steinberg, A first coherent structure in elastoinertial turbulence, arXiv:2006.06770.

[45] F. Waleffe, Three-Dimensional Coherent States in Plane Shear Flows, Phys. Rev. Lett. 81, 4140 (1998).

[46] A. Shekar and M. D. Graham, Exact coherent states with hairpin-like vortex structure in channel flow, J. Fluid Mech. 849, 76 (2018).

[47] A. Shekar, Structures and mechanisms in elastoinertial turbulence, Ph.D. thesis, University of WisconsinMadison, 2021.

[48] Y. Dubief, V. E. Terrapon, and J. Soria, On the mechanism of elasto-inertial turbulence, Phys. Fluids 25, 110817 (2013).

[49] A. S. Pereira, R. L. Thompson, and G. Mompean, Common features between the Newtonian laminarturbulent transition and the viscoelastic drag-reducing turbulence, J. Fluid Mech. 877, 405 (2019).

[50] Z. Wenhua, Z. Hongna, Y. Li, Y. Bo, and L. Fengchen, Role of elasto-inertial turbulence in viscoelastic drag-reducing turbulence, arXiv:2103.02359. 